\title{
Análise da importância da qualificação dos profissionais de saúde para o manejo do Transtorno de Espectro Autista (TEA)
}

Analysis of the importance of the qualification of health professionals for the management of Autistic Spectrum Disorder (ASD)

Análisis de la importancia de la cualificación de los profesionales de la salud para el tratamiento del Trastorno del Espectro Autista (TEA)

Thalita Ferreira Campos

ORCID: https://orcid.org/0000-0002-2850-6594 Faculdade Ciências Médicas da Paraíba/Afya, Brasil E-mail: thalitafcampos@gmail.com

Raíssa Guilherme Nunes Braga

ORCID: https://orcid.org/0000-0002-6290-3120 Faculdade Ciências Médicas da Paraíba/Afya, Brasil E-mail: raissanunesbraga@gmail.com

Levi Nogueira Moura

ORCID: https://orcid.org/0000-0003-4231-9338 Faculdade Ciências Médicas da Paraíba/Afya, Brasil E-mail: levi.nmoura10@gmail.com

Evando Raykaard Bessa de Queiroz ORCID: https://orcid.org/0000-0001-5117-7963 Faculdade Ciências Médicas da Paraíba/Afya, Brasil E-mail: evando1208@gmail.com

Tâmara Albuquerque Leite Guedes ORCID:https://orcid.org/0000-0003-2241-9032 Faculdade Ciências Médicas da Paraíba/Afya, Brasil E-mail: talguedes1@gmail.com

Letícia Hosana Araújo de Almeida

ORCID: https://orcid.org/0000-0003-4550-5654 Faculdade Ciências Médicas da Paraíba/Afya, Brasil E-mail: leticianutri2016@gmail.com

\begin{abstract}
Resumo
Objetivos: Este estudo tem como objetivo analisar a integralidade e a importância da qualificação dos profissionais da saúde, identificando a situação atual da capacitação profissional para o cuidado de pacientes com TEA no Brasil, bem como os problemas enfrentados nesse processo. Metodologia: Esta pesquisa consiste na investigação por meio de questionários quantificados e interpretados estatisticamente através do software Microsoft Excel, que buscou embasamento no Projeto de Pesquisa Muito Além do Autismo. Resultados e discussão: Os resultados mostram que 59\% dos entrevistados, durante sua formação profissional, tiveram algum contato com o tema do autismo. Ademais, no questionário a respeito da profundidade das informações abordadas sobre o tema durante a graduação, $27 \%$ responderam que o tema foi citado de forma superficial, isso mostra que há a necessidade de exploração maior sobre o autismo nas instituições de ensino. Conclusão: Na qualificação dos profissionais de saúde, atualmente, esse tema é abordado de maneira superficial nas graduações, dificultando o diagnóstico e intervenção precoce do transtorno.
\end{abstract}

Palavras-chave: Transtorno de espectro autista; Profissionais; Qualificação.

\begin{abstract}
Objectives: This study aims to analyze the integrality and the importance of the qualification of health professionals, identifying the current situation of professional training for the care of patients with ASD in Brazil, as well as the problems faced in this process. Methodology: This research consists of investigation through questionnaires, quantified and interpreted statistically using Microsoft Excel software, which sought to be based on the Research Project Muito Além do Autismo. Results and discussion: The results show that 59\% of the interviewees, during their professional training, had some contact with the topic of autism. Furthermore, in the questionnaire regarding the depth of information covered on the topic during graduation, $27 \%$ responded that the topic was mentioned in a superficial way, this shows that there is a need for further exploration of autism in educational institutions. Conclusion: Currently, in the
\end{abstract}


qualification of health professionals, this topic is superficially addressed in undergraduate courses, making the diagnosis and early intervention of the disorder difficult.

Keywords: Autism spectrum disorder; Professionals; Qualification.

\section{Resumen}

Objetivos: Este estudio tiene como objetivo analizar la integralidad y la importancia de la cualificación de los profesionales de la salud, identificando la situación actual de la formación profesional para el cuidados de los pacientes con TEA en Brasil, así como los problemas enfrentados en este proceso. Metodología: Esta investigación consiste en la indagación a través de cuestionarios, cuantificados e interpretados estadísticamente a través del software Microsoft Excel, que buscaba basarse en el Proyecto de Investigación mucho más allá del autismo. Resultados y discusión: Los resultados muestran que el 59\% de los encuestados, durante su formación profesional, tuvo algún contacto con el tema del autismo. Además, en el cuestionario relativo a la profundidad de la información cubierta sobre el tema durante la graduación, el $27 \%$ respondió que el tema fue mencionado de manera superficial, lo que demuestra que existe la necesidad de una mayor exploración del autismo en instituciones educativas. Conclusión: En la cualificación de los profesionales de la salud, actualmente, este tema se aborda de manera superficial en las graduaciones, lo que dificulta el diagnóstico y la intervención temprana del trastorno.

Palabras clave: Trastorno del espectro autista; Profesionales; Cualificación.

\section{Introdução}

O Transtorno de Espectro Autista (TEA) é um transtorno do desenvolvimento neural de curso crônico com sintomas de aparecimento precoce, que causam déficit na comunicação e interação social, bem como a formação de uma rotina repetitiva de comportamentos e atividades, com fixação demasiada em poucos interesses. Os sintomas do TEA causam prejuízos significativos ao desenvolvimento do portador, interferindo em seu funcionamento social, cognitivo e profissional (American Psychiatric Association, 2014).

O autismo é uma complexa temática vivida nas diversas esferas sociais, apresentando prejuízos de grande escala ao desenvolvimento do paciente, tornando a sua abordagem em meio acadêmico e profissional necessária, mas complicada (Martinoto, 2012).

O Autismo apresenta diversas nuances em relação aos sintomas que podem ser apresentados assim como na intensidade dos mesmos, podendo ser classificado como leve, moderado e severo. Diante dessa variação sintomática, mostra-se viável categorizá-lo como um transtorno de espectro, possibilitando a abrangência do TEA em sua totalidade. Desse modo, torna-se inviável homogeneizar o sujeito com autismo, uma vez que cada indivíduo apresenta sucintas distinções que fazem necessária a presença de um plano de cuidados singular. (Cunha, 2015)

O TEA é um transtorno complexo de difícil manejo, requerendo um plano de cuidado que necessita da participação, empenho e intervenção de uma equipe interdisciplinar. Isso comprova a necessidade do compartilhamento de ações entre as diversas áreas do conhecimento como a neuropediatria, psicologia, pedagogia, fonoaudiologia, nutrição, fisioterapia, entre outros. Além disso, quanto mais precoce a intervenção terapêutica mediada pela equipe, maiores são as possibilidades de desenvolvimento do autista.

Um estudo publicado em 2018, no qual estudantes do último ano do curso de Psicologia responderam questionários sobre o TEA, demonstrou que 295 estudantes apresentavam um baixo índice de acertos em relação a características importantes para o diagnóstico e tratamento do TEA, como: as abordagens terapêuticas para TEA; as escalas de rastreamento; os critérios para diagnóstico; a prevalência do transtorno; e as características que levantam suspeita de autismo. (Silva, Carvalho, Teixeira \& Paula, 2018).

Outro estudo, realizado em uma Instituição de Ensino Autista do interior paulista, evidenciou-se uma certa inabilidade e desconhecimento da maioria dos profissionais nessa área de atuação. O objetivo do trabalho foi identificar as principais dificuldades dos trabalhadores na interação com a criança autista com o intuito de formar um programa educativo com o viés de 
qualificá-los. Uma das queixas principais entre os participantes da pesquisa é a de que alguns não se sentem aptos para a função. (Montagner, Santiago \& Souza, 2007).

O profissional tem uma importância fundamental para atenuar as dificuldades que o autista e as famílias enfrentam, uma vez que um agente bem qualificado pode contribuir de forma significativa para impedir a evolução da doença, melhorando a qualidade de vida com ênfase no bem-estar físico, mental e social. Sob essa instância, as peculiaridades e manifestações das pessoas com autismo não podem se configurar como motivos de desistência nos aspectos pessoal, educacional e profissional. (Santos \& Vieira, 2017)

Com base nessa urgência para a intervenção terapêutica, é vital que haja a preparação profissional de qualidade para a formação de uma boa equipe interdisciplinar para tratamento do autismo. No entanto, embora haja essa necessidade de profissionais bem capacitados, a abordagem do TEA no meio acadêmico de um modo geral ainda é superficial, refletindo em mínimo conhecimento acerca da temática.

Esta pesquisa trata do nível de conhecimento dos profissionais e universitários da área de saúde em relação ao Transtorno de Espectro Autista (TEA). Sob tal ótica, percebe-se que o tema do autismo é abordado de maneira superficial no meio acadêmico. Consequentemente, há um déficit na formação desses profissionais decorrente da ausência de um espaço destinado para a temática nas grades curriculares pré-existentes, bem como a falta de uma especialização direcionada ao tratamento dos pacientes com TEA, embora haja uma demanda de pacientes autistas por profissionais qualificados.

Sendo assim, torna-se evidente a falta de abordagem de qualidade sobre o Transtorno de Espectro Autista (TEA) em cursos de atenção em saúde, tornando-se um fator preocupante para a formação dos futuros profissionais que entrarão em contato com pacientes autistas.

O trabalho tem como objetivo analisar a integralidade e a importância da qualificação dos profissionais da saúde no cuidado, no atendimento e no desenvolvimento biopsicossocial das crianças e dos adolescentes com autismo, identificando a situação atual da capacitação profissional no Brasil, bem como os problemas enfrentados diante da necessidade de uma abordagem qualificada e multidisciplinar da temática.

\section{Metodologia}

Este trabalho acatou as diretrizes e critérios estabelecidos na Resolução no 466 (2012) do Conselho Nacional de Saúde (CNS), após aprovação do Comitê de Ética em Pesquisa com o número do parecer 2.843.375, todos os dados foram coletados e analisados somente após receber a aprovação do sistema CEP-CONEP.

Este estudo segue o modelo retrospectivo, descritivo e transversal, de caráter exploratório. Ademais, foi utilizada uma abordagem quanti-qualitativa na coleta de dados.

Uma pesquisa retrospectiva se caracteriza por usar registros do passado que serão analisados até o presente, de modo que o fator causal ainda é existente. (Fletcher, 2003 como citado em Hochman, 2005).

A vertente qualitativa do estudo irá avaliar os fatores subjetivos observados na população estudada, por meio de questionários semi-estruturados.

Ao passo que os fenômenos objetivos e mensuráveis serão investigados por meio de questionários, quantificados e interpretados estatisticamente através do software Excel. Pois, de acordo com Fonseca (2002 como citado em Gerhardt et al., 2009 , p. 17), a pesquisa quantitativa pode utilizar-se da grafia matemática para elaborar relações de causa e consequência encontradas no estudo de um objeto, além de explicitar relações entre variáveis de forma objetiva e direta, dentre outras finalidades. Ademais, ainda segundo o autor supracitado, as contribuições da análise quantitativa podem ser potencializadas através do uso conjunto da análise qualitativa, permitindo ao pesquisador obter uma maior quantidade de informações do que se faria caso contrário. (Fonseca, 2002 como citado em Gerhardt et al., 2009, p. 35). 
A pesquisa foi realizada pelo projeto de extensão e pesquisa Muito Além do Autismo utilizando-se a ferramenta do Google Forms através de uma investigação por meio de questionários, quantificados e interpretados estatisticamente através do software Microsoft Excel. Questionário esse direcionado aos profissionais da saúde e aos estudantes de medicina.

Para analisar a percepção e o conhecimento de outros estudantes e de profissionais da saúde sobre a temática do autismo, será utilizado um questionário disponibilizado como formulário online através do Google Formulários, o formulário on-line foi preenchido após um Termo de Consentimento Livre e Esclarecido (TCLE).

Os dados referentes aos profissionais da saúde foram coletados via Google Formulário, contendo o questionário e o TCLE, que foram divulgados com os participantes via e-mail e redes sociais.

O caráter exploratório da pesquisa está relacionado à oportunidade de se familiarizar com a problemática do autismo através da óptica dos profissionais e como esses profissionais lidam sobre o assunto.

As análises quantitativas se configuram como ferramentas apropriadas para a apuração de opiniões e pensamentos explícitos pelos participantes da amostra em questão, podendo utilizar-se de questionários e outros instrumentos desse calibre. A partir dos dados obtidos, os pesquisadores podem generalizar e projetar essas informações como representativas da realidade da pesquisa.

O objetivo foi avaliar e projetar índices que possam ser comparados ao longo do processo de pesquisa, permitindo construir um histórico da informação.

Após a coleta, os dados quantitativos foram compilados em um banco de dados construído no Microsoft Excel (versão 2013) e foram avaliados através de estatística descritiva simples, tabelas e gráficos construídos no mesmo programa.

\section{Resultado}

A incidência de entrevistados que durante a sua formação profissional teve algum contato com o tema do autismo ou não tiveram contato, varia. Dos 324 entrevistados, 59\% disseram que tiveram algum contato, $40 \%$ disseram que não tiveram proximidade com o tema e $1 \%$ não responderam.

Figura 1 - Formação profissional e contato com o tema do autismo.

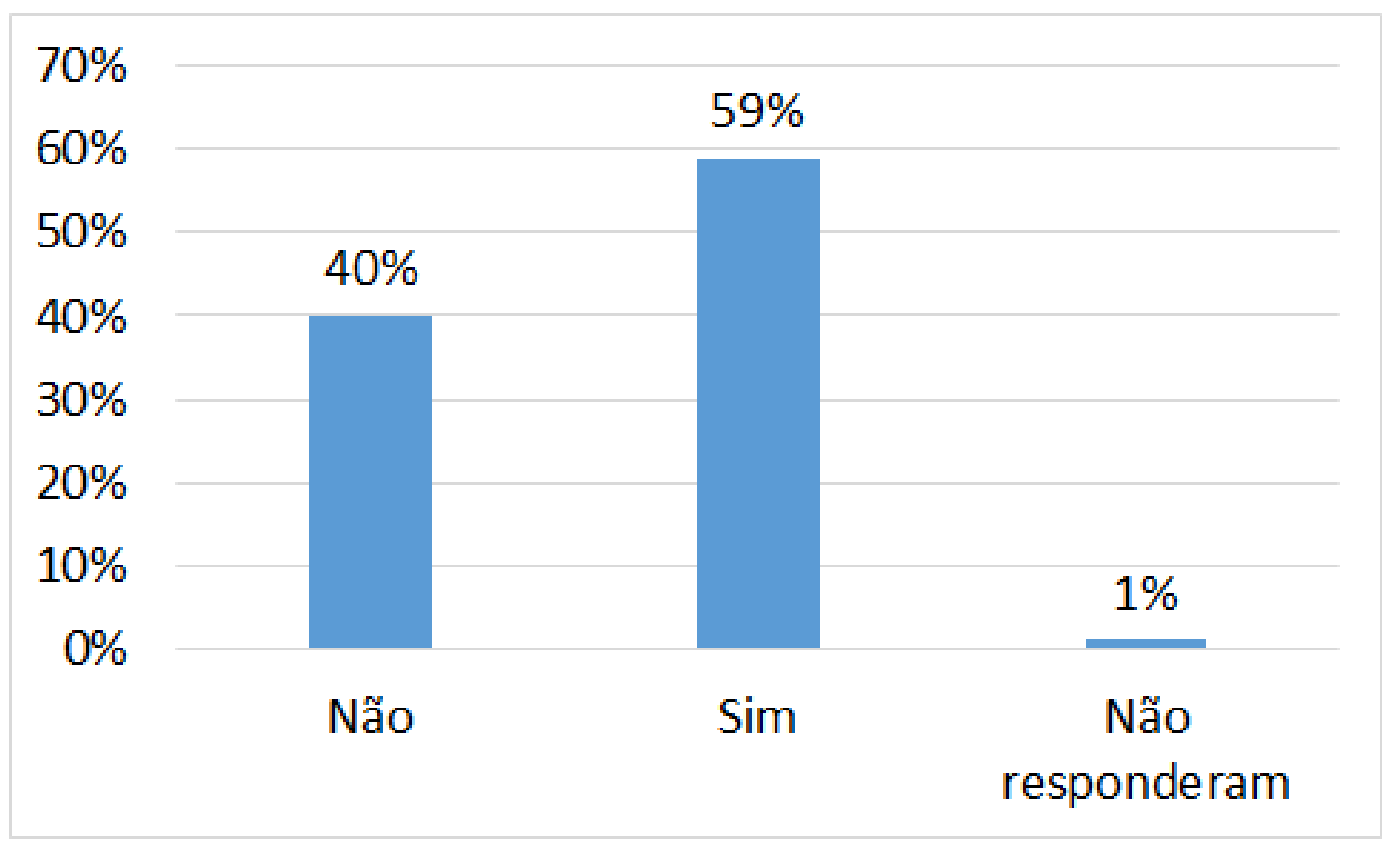

Fonte: Acervo do Projeto de pesquisa e extensão Muito Além do Autismo (2018). 
Há estudos que relatam sobre o retardo do diagnóstico devido à falta de esclarecimento sobre essa síndrome. Neste contexto, é essencial que os estudantes de medicina e a população como um todo conheça mais a fundo o transtorno do espectro autista para apontar o diagnóstico precoce. Segundo Mesquita e Pegoraro (2013), na medida em que atrasos na intervenção implicam na manutenção a longo prazo de comportamentos disruptivos, existe o interesse por diagnósticos e formas de intervenção precoces. Portanto, o debate no âmbito estudantil é necessário na tentativa de amenizar essa situação.

Segundo Silva et al. (2020) ainda existe uma carência de informação por parte tanto dos pais quanto dos profissionais sobre o autismo, o que dificulta no estabelecimento de estratégias de enfrentamento pela família e na aceitação do diagnóstico do TEA. Dessa forma, torna-se necessária a qualificação profissional sobre essa temática, facilitando e otimizando a abordagem profissional e familiar do paciente inserido no espectro.

A criança com autismo é relativamente capacitada, sendo um ser aprendiz com limitações e que precisa de um direcionamento no desenvolvimento da aprendizagem. Sabendo disso, o autista deve ser tratado como ser capaz, também deve ter trabalhos direcionados para suas restrições, vencendo qualquer dificuldade. Segundo Almeida e Albuquerque (2017), embora alguns autistas possuam comportamentos agressivos, essas pessoas devem viver da melhor maneira possível. Por isso, a importância dos profissionais possuírem informações adequadas sobre o diagnósticos do TEA e assim, os autistas terem uma melhor qualidade de vida.

Segundo Silva e Mulick (2009), cabe aos profissionais que lidam com o processo de diagnóstico serem cautelosos ao analisar determinados comportamentos e sintomas que refletem um quadro de autismo. Além disso, tendo em mente as manifestações multissistêmicas do TEA, o diagnóstico do espectro autista deve ser realizado de forma multidisciplinar para que haja uma ação conjunta no tratamento. Dessa forma, devem ser englobados, ao menos, um neuropediatra e um psicólogo com especialização em distúrbios do desenvolvimento. Sendo assim, esses profissionais contribuirão não só para o tratamento do quadro, mas também para a identificação do perfil médico, cognitivo e adaptativo da criança. Por isso, evidencia-se a relevância dos profissionais possuírem um melhor conhecimento sobre o TEA e assim conduzir da melhor forma o diagnóstico e o tratamento.

No questionário a respeito da profundidade das informações abordadas sobre o tema autismo durante a graduação, das 219 respostas obtidas $27 \%$ responderam que o tema foi citado de forma superficial, $11 \%$ a informação contemplou detalhes sobre o conceito de autismo, $8 \%$ a informação contemplou detalhes sobre diagnóstico, 5,85\% a universidade ensinou sobre as terapias vigentes para o autismo, 5,30\% foi contemplada a questão das políticas públicas vigentes ou em formação, 9,87\% foi contemplada a dimensão familiar envolvida, $13,52 \%$ foi contemplada dificuldades de inclusão social e uma porcentagem de 19,19\% não responderam à pergunta no questionário. Os resultados mostram a maior porcentagem dos dados à resposta em que o tema abordado na graduação não foi feito de maneira aprofundada, havendo a necessidade de exploração maior sobre autismo. 
Figura 2 - Profundidade das informações sobre o autismo.

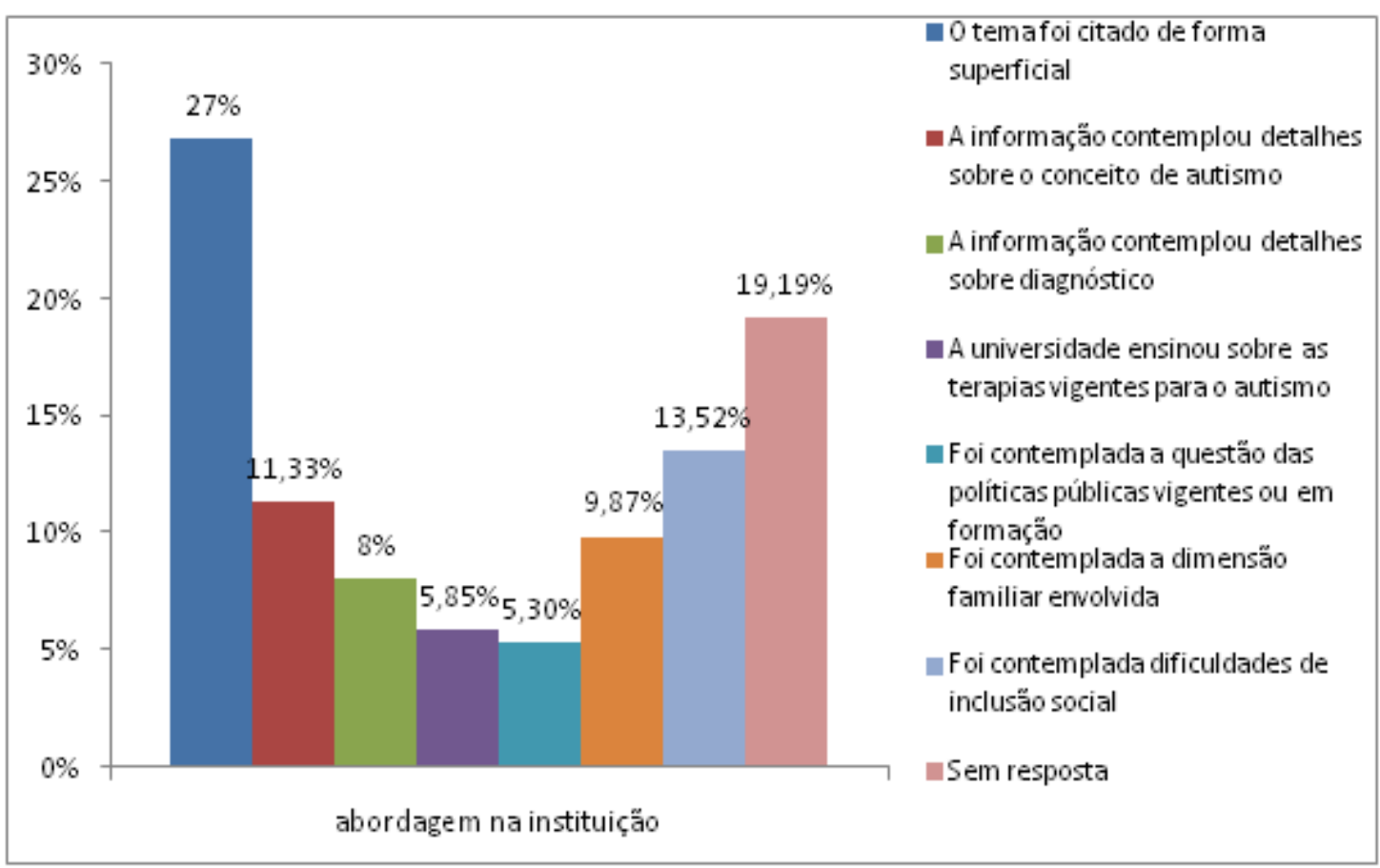

Fonte: Acervo do Projeto de pesquisa e extensão Muito Além do Autismo (2018).

Estudantes de enfermagem foram entrevistados sobre o transtorno do espectro do autismo e o trabalho publicado em 2019 evidenciou que esses acadêmicos possuíam pouco conhecimento sobre a temática. Além disso, é válido notar que as mídias e os meios de comunicação se mostraram importantes fontes de informação para o estudo do TEA por parte dos estudantes. (Ferreira \& Franzoi, 2019).

O transtorno do espectro do autismo na infância se tornou um obstáculo para o serviço público brasileiro devido à falta de informação e de profissionais com entendimento no que tange ao diagnóstico eficiente. Com o aparecimento dos primeiros casos do autismo, é notório a evolução do processo de conhecimento e que, consequentemente, auxilia em um melhor diagnóstico precoce possível, pois sabemos que cada pessoa no espectro possui uma progressão peculiar (Sousa \& Sousa, 2017 como citado em Silva, Lohmann, Costa \& Marchese, 2019, p. 8). Tal achado foi evidenciado por meio da pesquisa realizada, uma vez que os acadêmicos entrevistados apresentaram um conhecimento raso sobre a temática e, consequentemente, isso acarretaria no diagnóstico tardio causando restrições no seu desenvolvimento.

A falta de capacitação de alguns professores junto a falta de recursos escolares é frequente e tem-se notado que a inclusão está a cada dia mais frequente nas escolas, tornando necessário a busca pelo conhecimento a fim de receber e prepararse para estes alunos. (Octavio, Evaristo, Carvalho \& Fantacini, 2019).

\section{Discussão}

Estudos relatam que o diagnóstico precoce auxilia na intervenção da manutenção da redução de comportamentos disruptivos. Sendo assim, a Lei Berenice Piana 12.764 (2012), artigo $2^{\circ}$ alínea III, fala da importância de uma equipe multidisciplinar, do diagnóstico precoce e do acesso à medicação e nutrientes que são direitos dos autistas e devem ser todos sanados. Portanto, é indispensável que os profissionais conheçam esse transtorno para que haja um diagnóstico precoce, interações sociais e uma melhora na qualidade de vida dessas crianças. 
Ademais, pesquisas mostraram as desvantagens causadas diante da pouca informação e do pouco conhecimento sobre os pacientes com TEA, uma vez que a sociedade possui uma visão limitada e de certa forma preconceituosa sobre o espectro e o diagnóstico pode passar despercebido, afetando no processo de tratamento e dificultando no desenvolvimento da pessoa com TEA. Por isso, são necessários mais estudos, pesquisas e informações sobre o autismo com o objetivo de proporcionar uma melhor qualidade de vida através de um diagnóstico precoce. (Dartora, Mendieta \& Franchini, 2014 como citado em Ferreira \& Franzoi, 2019, p. 51).

\section{Conclusão}

Os resultados mostram, de modo geral, que 59\% dos entrevistados, durante sua formação profissional, tiveram algum contato com o tema do autismo. Além disso, 27\% responderam que o tema foi citado de forma superficial na graduação, mostrando que há a necessidade de exploração maior sobre autismo.

A qualificação dos profissionais de saúde atualmente toma-se conhecimento do tema, porém difere na profundidade do assunto tendo em vista o grau de estudo da temática nas graduações, as quais predominam o ensino escasso no domínio e abordagem do conteúdo, havendo a necessidade de proximidade de assuntos referentes à temática, possibilitando o diagnóstico e intervenção precoce do transtorno.

Assim sendo, quanto mais cedo for detectado o transtorno, mais contribuições e mais qualidade de vida poderão ser disponibilizadas para o autista. Contribuições estas que reduzem os efeitos disruptivos e os distúrbios neuropsicomotores, ajudando nos processos de comunicação e de interação social. Para que esse objetivo seja alcançado, é pertinente que as instituições de ensino em saúde incentivem os alunos a buscarem mais conhecimento nessa temática por meio de atividades extracurriculares, aulas aprofundadas e cursos preparatórios para os futuros profissionais de saúde no que diz respeito ao diagnóstico, tratamento, encaminhamento correto e cuidados multidisciplinares. Portanto, quanto mais cedo for abordado na comunidade acadêmica, mais os profissionais de saúde estarão capacitados para identificar o TEA.

\section{Referências}

Almeida, C. M., \& Albuquerque, K. (2017). Autismo: Importância da Detecção e Intervenção Precoces. Revista Científica Multidisciplinar Núcleo do Conhecimento. $1,488-502$.

American Psychiatric Association. (2014). Manual diagnóstico e estatístico de transtornos mentais: DSM-5: Artmed.

Cunha, E. (2015). Autismo e inclusão: psicopedagogia práticas educativas na escola e na família (6a ed.): Wak Ed.

Favoretto, N. C., \& Lamonica, D. A. C. (2014). Conhecimentos e necessidades dos professores em relação aos transtornos do espectro autístico. Rev Bras Ed Esp. 20(1), 103-116.

Ferreira, A. C. S. S., \& Franzoi, M. A. H. (2019). Conhecimento de estudantes de enfermagem sobre os transtornos autísticos. Revista de enfermagem UFPE. 13(1), 51-60.

Freire, P. (1992). A importância do ato de ler: em três artigos que se completam: Cortez: Autores Associados.

Gerhardt, T. E., \& Silveira, D. T. (2009). Métodos de pesquisa (1a ed.): Editora da UFRGS, 17-35.

Hochman, B., et al. (2005). Desenhos de pesquisa. Acta Cir. Bras., São Paulo. 20 (supl. 2), 2-9. 10.1590/S0102-86502005000800002.

Instituto Federal da Paraíba. (2017). Cartilha institucional: Conhecendo o transtorno do espectro autista. João Pessoa: Autor. https://www.ifpb.edu.br/sousa/noticias/2017/04/transtorno-do-espectro-do-autismo-e-tema-de-acao-educativa/cartilha-institucional-do-transtorno-do-espectroautista.pdf/

Lei $n^{\circ}$ 12.764, de 27 de dezembro de 2012. Institui a Política Nacional de Proteção dos Direitos da Pessoa com Transtorno do Espectro Autista, e altera o $\$ 3^{\circ}$ do art. 98 da Lei $n^{\circ} 8.112$, de 11 de dezembro de 1990. http://www.planalto.gov.br/ccivil_03/_ato2011-2014/2012/lei/112764.htm

Losapio, M. F., \& Pondé, M. P. (2008). Tradução para o português da escala M-CHAT para rastreamento precoce de autismo. Rev Psiquiatr Rio Gd Sul. 30(3), 221-229.

Martinoto, L. B. (2012). A importância da qualificação do profissional da educação infantil no atendimento de crianças com autismo. Revista Vento e Movimento. $1(1), 6-20$. 
Research, Society and Development, v. 10, n. 6, e32910615667, 2021

(CC BY 4.0) | ISSN 2525-3409 | DOI: http://dx.doi.org/10.33448/rsd-v10i6.15667

Mesquita, W. S., \& Pegoraro, R. F. (2013). Diagnóstico e tratamento do transtorno autístico em publicações brasileiras: revisão de literatura. J Health Sci Inst. 31(3), 324-329.

Montagner, J., Santiago, È., \& Sousa, M. G. G. (2007). Dificuldades de interação dos profissionais com as crianças autistas de uma instituição educacional de autismo. Arq Ciênc Saúde. 14(3), 169-174.

Octavio, A. J. M., Alves, A. L. E., Carvalho, B. M., \& Fantacini, R. A. F. (2019). A inclusão do aluno com transtorno do espectro autista na educação infantil. Research, Society and Development. 8(1), 10. 10.33448/rsd-v8i1.635.

Resolução n. ${ }^{\circ}$ 466, de 12 de dezembro de 2021. Dispõe sobre as diretrizes e normas regulamentadoras de pesquisas envolvendo seres humanos. https://bvsms.saude.gov.br/bvs/saudelegis/cns/2013/res0466_12_12_2012.html

Santos, R. K., \& Vieira, A. M. E. C. S. (2017). Transtorno do espectro autista (TEA): do reconhecimento à inclusão no âmbito educacional. Revista Includere. $3(1), 219-232$.

Schmidt, C. (2017). Transtorno do Espectro Autista: Onde estamos e para onde vamos. Psicologia em Estudo. 22(2).

Silva, C. O., Oliveira, S. A., Silva, W. C., Mendes, R. C., Miranda, L. S. C., Melo, K. C., Silva, R. A., Oliveira, T. M. P., Oliveira, C. J. P., \& Santos, M. E. J. (2020). Benefits in using early interventio in children with autista spectrum disorder (ASD): an integrative review. Research, Society and Development. 9(7), $1-8$.

Silva, L. V., Carvalho, F. A., Teixeira, M.C.V., \& Paula, C. S. (2018). Formação do psicólogo sobre autismo: estudo transversal com estudantes de graduação. Revista Psicologia: Teoria e Prática. 3(20), 138-152.

Silva, M., \& Mulick, J. A. (2009). Diagnosticando o Transtorno Autista: Aspectos Fundamentais e Considerações Práticas. Psicologia Ciência e Profissão. 29(1), 116-131.

Silva, S. A., Lohmann, P. M., Costa, A. E. K., \& Marchese, C. (2019). Conhecimento da equipe interprofissional acerca do autismo infantil. Res., Soc. Dev. $8(9), 8$.

Tamanaha, A. C., Perissinoto, J., \& Brasilia, M. C. (2008). Uma breve revisão histórica sobre a construção dos conceitos do Autismo Infantil e da síndrome de Asperger. Rev Soc Bras Fonoaudiol. 13(3), 296-299. 